\title{
Dental Arch Morphology as a Predictor of Sleep Disordered Breathing
}

\author{
Oommen Nainan ${ }^{1 *}$, Balakrishnan Jayan², Rajat Mitra ${ }^{3}$, Snehashish Ghosh ${ }^{4}$, Sukhbir Singh Chopra5 \\ Manish Mukherjee 6 \\ ${ }^{1}$ Graded Specialist-Orthodontics \& Dentofacial Orthopedics, Naval Institute of Dental Sciences,INHS Asvini Campus, Colaba, Mumbai-400005, \\ Maharashtra State, India \\ ${ }^{2}$ Consultant-Orthodontics \& Dentofacial Orthopedics, Army Dental Centre (Research \& Referral), Delhi Cantt, New Delhi-110010, India \\ ${ }^{3}$ Classified Specialist-Orthodontics \& Dentofacial Orthopedics, Commanding Officer \& Corps Dental Adviser, 1 Corps Dental Unit, C/o 56 APO, India \\ ${ }^{4}$ Classified Specialist-Orthodontics \& Dentofacial Orthopedics, Military Dental Centre, Barrackpore, West Bengal, India \\ ${ }^{5}$ Senior Specialist-Orthodontics \& Dentofacial Orthopedics, Army Dental Centre (Research \& Referral), Delhi Cantt, New Delhi-110010, India \\ ${ }^{6}$ Classified Specialist-Periodontology, Commanding Officer, Military Dental Centre, Meerut, India
}

\begin{abstract}
Objective: There is limited data available regarding dental arch morphology and its association with Sleep Disordered Breathing (SDB). The objective of the study was to compare the dental arch morphology of patients with Obstructive Sleep Apnoea-Hypopnoea Syndrome (OSAHS) and of those without any symptoms of SDB.

Patients/Methods: A total of 600 subjects were included in the study and were divided into two groups of 300 subjects each (390 men and 210 women); Group 1: Patient group; Group 2: Control group. The test and control subjects were well matched for age and gender. A comparative cross-sectional study design was employed and specific measurements were made on dental study models with digital calipers to ensure precise and accurate measurement of anatomic distances.

Results: Analyses were able to detect statistically significant changes $(P<0.05)$ in maxillary and mandibular dental arch and alveolar width dimensions. The prevalence of recorded malocclusions in the OSA group in terms of molar/canine relation was also high, with most of the test subjects having an Angle's Class II malocclusion.

Conclusions: Indian adults with OSA have a dental arch morphology, which is different from that of normal subjects, of similar age and gender. It is necessary to give due consideration to the evaluation of the dental arch morphology of patients with SDB before the appropriate treatment is offered.

Keywords: dental arch dimensions, study model analysis, obstructive sleep apnoea, malocclusion, orthodontist, palatal depth, polysomnography, mouth breathing
\end{abstract}

\section{INTRODUCTION}

Obstructive sleep apnoea-hypopnoea syndrome (OSAHS) is a disorder characterized by repetitive

*Correspondence: droommennainanayahoo.in

Surgeon Commander (Dr) Oommen Nainan, Graded SpecialistOrthodontics \& Dentofacial Orthopedics, Naval Institute Of Dental Sciences, INHS Asvini Campus, Colaba. Mumbai-400005, Maharashtra State, India Contact number: +919869860710

\section{Sleep and Hypnosis \\ Submit your manuscript at www.sleepandhypnosis.org}

episodes of pharyngeal airway collapse resulting in decreased airflow during sleep and can adversely impact longevity and quality of life (Fleisher and Krieger, 2007). A study conducted in an Indian population estimated the prevalence of sleep-disordered breathing (SDB) among middle-aged urban Indian men to be at $19.5 \%$ and that of OSAHS to be $7.5 \%$ (Udwadia, Doshi, Lonkar, \& Singh, 2004). There is limited data available regarding dental arch morphology and its association with OSA, especially in comparison with the numerous articles that can be found concerning skeletal changes that are found in 
patients with OSA. Therefore a study was carried out to evaluate the dental arch characteristics in Indian adults with OSA and compare the findings with those without OSA. The following study was taken up with the aim to test the null hypothesis that there is no difference in dental arch features and dental arch morphology in Indian adults with and without SDB. The objective of the study was to compare the dental arch morphology of patients with OSA and of those without any symptoms of SDB.

\section{MATERIALS AND METHODS}

This multi-disciplinary study was carried out at the Division of Orthodontics of a tertiary care dental establishment. Power and sample size software was used to calculate the sample size based on comparing two means - dental arch of OSA and control subjects (Dupont and Plummer, 1997). To detect a difference of $1 \mathrm{~mm}$ (which is considered to be clinically significant) with $80 \%$ power, $a=0.05$ and a ratio between two groups of $1: 1$, 600 adult Indians aged 18 years and above were recruited for this study.Data were collected in a time span of two years. A comparative cross-sectional study design was employed. The subjects were divided into two groups as under:

Patients:The patient sample consisted of 300 randomly selected polysomnography(PSG) diagnosed OSA patients referred by Department of Respiratory Medicine of 5 tertiary care medical establishments, for the analysis of dental arch morphology. Each of the subjects in this group underwent a level-I overnight PSG (Chervin, Murman, Malow, \& Totten, 1999). The patients who satisfied the following criterion were included: polysomnographic evidence of OSA [apnoea/hypopnoea index (AHI) of $\geq 5 /$ hour of sleep] and presence of at least ten teeth in each arch.

Control subjects: The control group consisted of 300 randomly selected adults who matched to the best extent possible, with the patient sample for age, gender, height and weight. A total of 487 subjects were screened with a questionnaire, before finalizing 300 controls.
Excessive daytime sleepiness(EDS) was assessed with the Epworth Sleepiness Scale (ESS) (WJohns, 1993). The patients who satisfied the following criterion were included: Class I occlusion;ESS score < 9;no reported snoring by family members and presence of at least ten teeth in each arch.

The exclusion criteria for both groups were:edentulous subjects; hypothyroidism, history of orthodontic treatment/orthognathic surgery/craniofacial deformity. The research protocols were reviewed and approved by the Institutional Ethical Committee of the hospital. Informed consent was obtained from each participant,after explaining the nature and purpose of the study.

\section{Method}

Data collection was divided into three sections as follows:

a. Medical and sleep history.

b.Clinical examination with anthropomorphic recordings of the subject's height,weight and neck circumference.Body mass index (BMI) was used as a measure of the obesity level of a subject (Revicki and Israel, 1986). Overweight condition was diagnosed if BMl was $>25$ and $\leq 30 \mathrm{~kg} / \mathrm{m}^{2}$.

c. Study model analysis: Measurements were taken from the maxillary and mandibular study models using a pair of digital calipers (Workzone, Globaltronics $\mathrm{GmbH} \&$ \& Co, Germany), with a resolution of $0.01 \mathrm{~mm}$ (Figure 1).

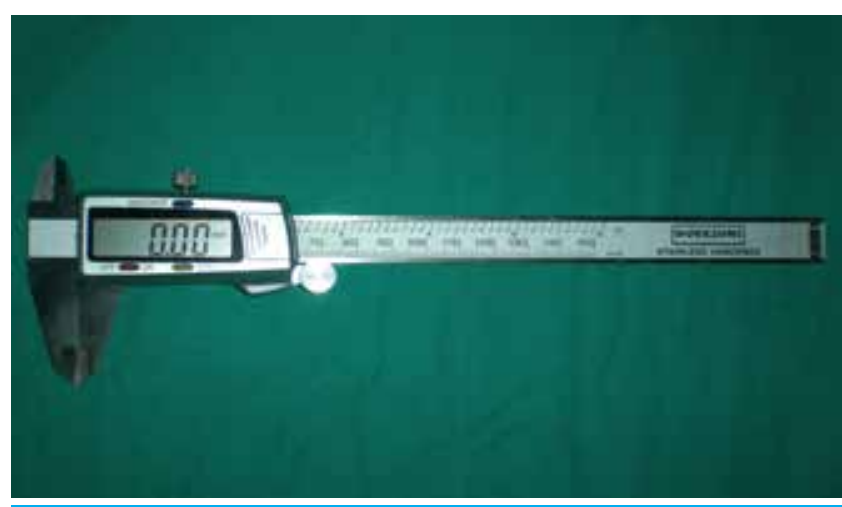

Figure 1: Digital Vernier Caliper 


\section{ORTHODONTIC EVALUATION}

\section{(a) Clinical examination}

An orthodontic assessment of the occlusion was made in addition to periodontal and soft tissue examination.Visibility of pharyngeal structures was noted and evaluation for air space between soft palate and tongue was done using the modified Mallampati classification (MMC) (Zonato, Bittencourt, Martinho, Junior, Gregório, \& Tufik, 2003).

\section{(b) Impressions and Study model analysis}

The upper and lower impressions of subjects in both the groups were made and study models prepared according to professional standards.The methodology validated by previous studies, was used to make the following measurements on the study models (Sayin and Turkkahraman, 2004) (Figures 2 \& 3):

\section{Maxillary intercanine width[UCW]:Distance}



Figure 2: Arch width measurements on maxillary study model

between cusp tips of maxillary right and left canines.

2. Maxillary interpremolar width I[UPW I]:Distance between buccal cusp tips of maxillary right and left first premolars.

3. Maxillary interpremolar width II[UPW II]:Distance between buccal cusp tips of maxillary right and left second premolars.

4. Maxillary intermolar width I[UM I]:Distance between the mesiobuccal cusp tips of maxillary right and left first molars.

5. Maxillary intermolar width II[UM II]:Distance between central fossae of maxillary right and left first molars.

\section{Maxillary interalveolar width [UAW]:Distance} between mucogingival junctions above the mesiobuccal cusp tips of maxillary right and left first molars.

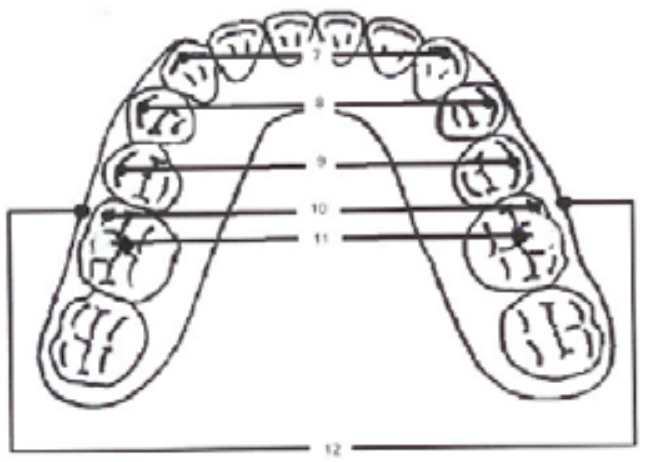

Figure 3: Arch width measurements on mandibular study model

7. Mandibular intercanine width[LCW]:Distance between cusp tips of mandibular right and left canines.

8. Mandibular interpremolar width I[LPW I]:Distance between buccal cusp tips of mandibular right and left first premolars.

9. Mandibular interpremolar width II[LPW II]:Distance between buccal cusp tips of mandibular right and left second premolars.

10. Mandibular intermolar width I[LM I]:Distance between mesiobuccal cusp tips of mandibular right and left first molars.

11. Mandibular intermolar width II[LM II]:Distance between central fossae of mandibular right and left first molars.

12. Mandibular interalveolar width[LAW]:Distance between mucogingival junctions below buccal grooves of mandibular right and left first molars. In subjects where teeth were absent, linear measurement was made to the apex of the edentulous ridge bisecting a line drawn from centroids of abutment teeth.

13. Overjet [OJ]:The horizontal distance from edge of maxillary central incisor to labial surface of mandibular central incisor along a line parallel to the occlusal plane with teeth in centric occlusion.

14. Overbite [OB]:The perpendicular distance from the lower to upper central incisor edge(Thilander, Pena, Infante, Parada, \& de Mayorga, 2001). 
15. Angle's class of malocclusion [AC]:Sagittal molar relationship was classified Class I, Class II Division I, Class II Division II and Class III.

16. Depth of palate [PH]:To standardize measurement of palatal depth, models were trimmed until the distal contact point of upper first molars showed up on the edge.Distance from the mid-deepest part of the palate to the line connecting the left and right distolingual cusp tips of upper first molars was taken as palatal depth(Figure 4).

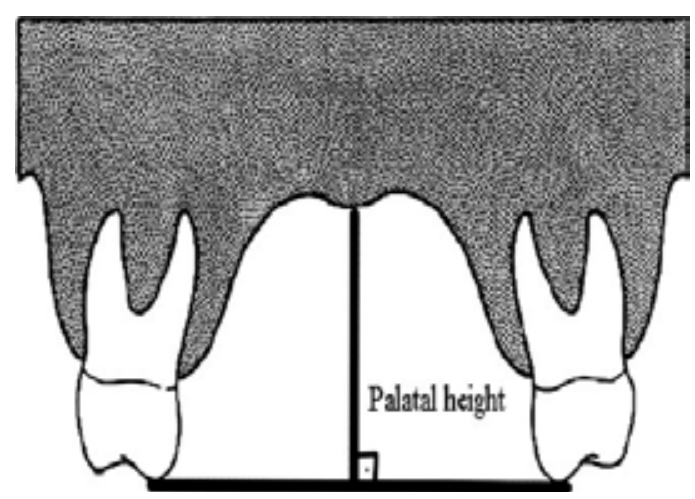

Figure 4 : Measurement of palatal height/depth

\section{Data compilation and statistical analysis}

In order to calculate the error of method, the measurements of 60 randomly selected casts was repeated on separate occasions with a 2-week interval.All measurements obtained were expressed as mean \pm standard deviation(SD) and compared using analysis of variance(ANOVA).The differences between groups were established using regression coefficient equation. P value less than or equal to 0.05 was considered statistically significant. Intra-rater reliability was measured by intraclass correlation coefficients (ICC) for linear dental arch measurements and Cohen's kappa coefficients for malocclusion classification. As the two groups were gender and age matched, the material was regarded as a paired sample when analysing malocclusion prevalences. A binomial test was used to examine the malocclusion prevalence of the paired sample. The data was analysed using the MATLAB version 1.0 and Excel 2007.

\section{RESULTS}

\section{Characteristics Of Patients And Controls}

Each group consisted of 190 men and 110 women. The ages ranged from 32 to 68 years. The mean age was $53.6 \pm 9.42$ years. Test and control subjects were well matched for age and gender. While the two groups had no significant difference in terms of height, OSA patients were found to be significantly heavier with a resulting statistically significant increase in BMI, as shown in Table 1. The patients in the test group also had a statistically significant increase in neck circumference and a higher Mallampati score.

\section{Sleep data of patients}

All patients in the test group had varying degree of OSA, as confirmed by overnight PSG. The AHI ranged from 5.6 to 86.4 events/hour with a mean of $42.04 \pm 26.14$ events/hour.

Table 1: Comparison of mean of general physical characteristics between test and control groups

\begin{tabular}{|c|c|c|c|c|c|c|c|c|}
\hline \multirow[b]{2}{*}{ S no } & \multirow[b]{2}{*}{ Parameter } & \multicolumn{2}{|c|}{$\begin{array}{l}\text { Test Group } \\
(n 1=300)\end{array}$} & \multicolumn{2}{|c|}{$\begin{array}{l}\text { Control Group } \\
(n 2=300)\end{array}$} & \multirow[b]{2}{*}{$\underset{(n 1+n 2-2)}{d f}$} & \multirow[b]{2}{*}{ P Value } & \multirow[b]{2}{*}{$P$ value $(<0.05)$} \\
\hline & & Mean & SD & Mean & SD & & & \\
\hline 1 & $\mathrm{Ht}$ in mtrs & 1.64 & 0.01 & 1.642 & 0.09 & 598 & 1 & 1 \\
\hline 2 & Wt in kgs & 80.1 & 12.5 & 67.13 & 6.78 & 598 & 0.00019 & $<0.0001^{*}$ \\
\hline 3 & BMI $\left(\mathrm{kg} / \mathrm{m}^{2}\right)$ & 29.47 & 4.57 & 24.84 & 1.87 & 598 & 0.00016 & $<0.0001^{\star}$ \\
\hline 4 & $\mathrm{NC}$ in $\mathrm{cms}$ & 39.8 & 3.16 & 36.1 & 2.59 & 598 & 0.00012 & $<0.0001^{\star}$ \\
\hline 5 & ESS & 13 & 2.85 & 3.26 & 0.94 & 598 & 0.00018 & $<0.0001^{\star}$ \\
\hline 6 & $\mathrm{AHI}$ & 42.04 & 26.14 & & & 299 & & \\
\hline
\end{tabular}

Ht- height; Wt- weight; BMI- body mass index; NC- neck circumference;

ESS- Epworth sleepiness scale; AHI-apnoea hypopnoea index,

k- number of cell means

$* P \leq 0.05=$ statistically significant. 


\section{Questionnaire data of patients and controls}

All 600 subjects completed the questionnaire. Among the controls, the ESS ranged from 2 to 5 with a mean of $3.26 \pm 0.94$.In the test group, the ESS recorded a statistically significant increase and ranged from 9 to 20 with a mean of $13 \pm 2.85$. Most of the test subjects also rated their sleep quality as short, interrupted and restless.

\section{DENTAL ARCH CHARACTERISTICS Maxillary arch morphology}

The comparison of mean intraoral values between OSA and controls is summarised in Table 2.The differences between the dental arch measurements were studied using regression coefficient equation. While overbite was found to be reduced in subjects with OSA, analyses failed to detect statistically significant differences when overjet and overbite of OSA and controls were compared.35 OSA patients had an edge to edge incisal relationship,while 8 patients had an anterior open bite of $1-2 \mathrm{~mm}$. None of the patients had a transverse discrepancy/crossbite. Statistically significant differences were found between the groups for the measurements of palatal depth. The mean maxillary arch width was smaller in the group of OSA subjects when measured at the canines and interalveolar area between first molars, while the width in all other regions was larger.Comparison of the upper study models for size differences indicated disproportional changes, with the mean OSA upper arch being $3 \%$ narrower in the canine region and $1 \%$ narrower in interalveolar width, $2 \%$ wider in the premolar region and $3 \%$ wider in the molar region. In view of these findings, analyses were able to detect statistically significant changes for all dimensions, except for maxillary interalveolar width and maxillary interpremolar width between second premolars.

\section{Mandibular arch morphology}

The comparison of mean intraoral values for the lower arch is summarised in Table 2.Statistically significant differences were found between the groups for inter canine width and inter premolar width between second premolars.Analyses also indicated disproportional

Table 2: Comparison of mean of intraoral measurements between test and control groups

\begin{tabular}{|c|c|c|c|c|c|c|c|c|c|}
\hline \multirow[b]{2}{*}{ S no } & \multirow[b]{2}{*}{ Parameter } & \multicolumn{2}{|c|}{$\begin{array}{c}\text { Test Group } \\
(n=300)\end{array}$} & \multicolumn{2}{|c|}{$\begin{array}{l}\text { Control Group } \\
(n=300)\end{array}$} & \multirow[b]{2}{*}{ df } & \multirow[b]{2}{*}{$\begin{array}{l}\text { Regression } \\
\text { coefficient }\end{array}$} & \multirow[b]{2}{*}{$\begin{array}{l}P \text { value } \\
(<0.05)\end{array}$} & \multirow[b]{2}{*}{$\begin{array}{l}R \text { value } \\
(<0.01)\end{array}$} \\
\hline & & Mean & SD & Mean & SD & & & & \\
\hline 1 & UCW & 34.47 & 2.48 & 35.39 & 2.49 & 1 & 0.155 & $<0.05^{\star}$ & $<0.01 * *$ \\
\hline 2 & UPW-I & 42.32 & 3.11 & 41.47 & 2.58 & 1 & 0.394 & $<0.05^{\star}$ & $<0.01 * *$ \\
\hline 3 & UPW-II & 47.62 & 3.48 & 46.07 & 3.29 & 1 & -0.286 & 0.0815 & $\geq 0.01$ \\
\hline 4 & UM-I & 53.05 & 3.57 & 51.58 & 3.26 & 1 & 0.061 & $<0.05^{\star}$ & $<0.01 * *$ \\
\hline 5 & UM-II & 47.97 & 3.4 & 46.13 & 3.22 & 1 & -0.119 & $<0.05^{\star}$ & $<0.01^{\star *}$ \\
\hline 6 & UAW & 57.12 & 3.1 & 57.51 & 2.36 & 1 & 0.158 & 0.585 & $\geq 0.01$ \\
\hline 7 & LCW & 25.73 & 2.64 & 26.67 & 2.07 & 1 & -0.097 & $<0.05^{\star}$ & $<0.01^{* *}$ \\
\hline 8 & LPW-I & 26.67 & 2.63 & 34.19 & 2.65 & 1 & -0.121 & 0.142 & $\geq 0.01$ \\
\hline 9 & LPW-II & 41.12 & 3.58 & 39.1 & 3.51 & 1 & 0.137 & $<0.05^{\star}$ & $<0.01 * *$ \\
\hline 10 & LM-I & 46.51 & 3.93 & 44.48 & 2.87 & 1 & -1.875 & 0.208 & $\geq 0.01$ \\
\hline 11 & LM-II & 42.81 & 3.41 & 40.64 & 2.71 & 1 & 1.351 & 0.151 & $\geq 0.01$ \\
\hline 12 & LAW & 54.4 & 3.47 & 53.2 & 3.26 & 1 & 0.034 & 0.823 & $\geq 0.01$ \\
\hline 13 & OJ & 1.97 & 1.92 & 2.8 & 1.76 & 1 & 0.06 & 0.086 & $\geq 0.01$ \\
\hline 14 & $\mathrm{OB}$ & 1.56 & 1.28 & 2.03 & 0.76 & 1 & 0.359 & 0.089 & $\geq 0.01$ \\
\hline 15 & $\mathrm{PH}$ & 24.1 & 1.74 & 20.75 & 2.01 & 1 & 0.043 & $<0.05^{\star}$ & $<0.01^{* *}$ \\
\hline
\end{tabular}

Note. UCW-maxillary intercanine width; UPW I-maxillary interpremolar width I;UPW II-maxillary interpremolar width II ;

UM I-maxillary intermolar width I ; UM II-maxillary intermolar width II ;UAW- maxillary interalveolar width;

LCW- mandibular intercanine width; LPW I- mandibular interpremolar width I ;

LPW II-mandibular interpremolar width II ;LM I- mandibular intermolar width I;

LM II-mandibular intermolar width II; LAW- mandibular interalveolar width ; OJ- overjet; OB-overbite;

$\mathrm{PH}$-depth of palate; AC-Angle's class of malocclusion.

${ }^{*} \mathrm{P} \leq 0.05$ = statistically significant.

${ }^{* *} \mathrm{R}<0.01=$ statistically significant. 
changes with narrowing in the canine region, $5 \%$ widening in the premolar region, $4 \%$ widening in the molar region and $5 \%$ widening in interalveolar width with respect to test group.

The prevalence of recorded malocclusions in the OSA group in terms of molar/canine relation was high, with 180 (60\%) of the 60 test subjects having an Angle's Class II malocclusion. Sixteen patients were also found to have a Class III molar relation. According to independent samples t-test, statistically significant differences were found in maxillary and mandibular dental arch and alveolar width dimensions. Thus, the null hypothesis was rejected.

\section{DISCUSSION}

India is the seventh-largest country by geographical area and second-most populous country with over 1.2 billion people. No data are available on the dental arch dimensions of patients with OSA from the Indian subcontinent and a Medline search confirms that this is the first study evaluating the dental arch morphology in PSG confirmed patients of OSA, from India.

The recognition and treatment of snoring and OSA are two medical areas where dentistry can play a valuable role.Dentists in general and Orthodontists in particular now have an ever increasing role in the recognition of a patient who may be at risk for a sleep disorder.Clinicians have used various factors to predict a person's risk of having OSA, based on their demographics, symptoms and body-mass index. Using these factors, they have been able to correctly identify $76 \%-96 \%$ of patients who have OSA and $13 \%-54 \%$ of patients who do not have OSA (Lee, Chan, Grunstein, \& Cistulli, 2009).

The findings of previous studies have led to the hypothesis that the size and shape of the dental arches could have a role to play in the development of OSA.The dental arch form is initially shaped according to the configuration of the supporting bone and following the eruption of the teeth, by the circum-oral musculature and intra-oral functional forces (Harris and Johnson, 1991). Studies have indicated that the shape and dimensions of the maxilla were important determinants of nasal airway resistance and OSA severity in patients with Marfan's syndrome (Cistulli, Richards, Palmisano, Unger, BerthonJones, \& Sullivan, 1996).

The results of this current study in Indian patients indicate that $\mathrm{BMI}$ and neck circumference are significantly greater for the OSA group when compared with the control group and showed a statistically significant correlation with severity of OSA.Contrary to the commonly held belief that OSA in Asians is found more frequently in non-obese subjects,it was observed that severe OSA is seen in obese Indian patients (Lam, Ip, Tench, \& Ryan, 2005). This difference in findings could be because of environmental variation, as the majority of previously cited studies were undertaken on Asian subjects living in western societies. These findings support the view that obesity is a significant risk factor for OSA in Indian adults,which is in complete agreement with the findings obtained in other populations (Ferguson, Ono, Lowe, Ryan, \& Fleetham, 1995).

The strength of the present study is the inclusion of a relatively large group of Indian patients with OSA, who underwent a limited hospital-based sleep study, matched with control subjects for age and gender.While every attempt was made to match patients with OSA to control subjects for age and BMI, the OSA group was found to be more obese. This study highlights the importance of matching subjects for age to prevent any age-related morphological changes from confounding the results, with previous studies typically selecting controls who were significantly younger (Battagel, Johal, \& Kotecha, 2000). In accordance with other reported studies, OSA was found to be more common in men than women and increasing with age.

The present study indicates that Indian patients with SDB in general and OSA in particular have differences in dental arch dimensions. Measurements were made on study models, by means of digital vernier callipers. Although some new methods like geometric morphometrics provide excellent possibilities for morphological analysis, distance measurements on dental casts were calculated because most clinicians are familiar with the method used, is easily reproducible and cost effective. Comparison of the upper arch study models for 
size differences indicated disproportional changes, with the mean upper arch width of the OSA group being narrower in the canine region and in interalveolar width while being wider in the premolar and molar regions. Differences between patients and controls varied from 0.45 to $1.47 \mathrm{~mm}$ in all the above measurements. Maxillary arch width was constricted in the anterior region of the arch, probably due to mouth breathing and an altered position of the tongue, which changes the muscular balance between the tongue and the cheeks. A narrower upper anterior dental arch may also be explained by the sleeping position. It has been suggested that a supine sleeping posture is related to a higher $\mathrm{AHI}$ and that a prolonged supine sleeping position correlates with reduced intermaxillary width (Pirilá, Tahvanainen, Huggare, Nieminen, \& Löppönen, 1995). Since the patients and controls were exactly matched for age, it is likely that the changes in the dental arch could be a reflection of the underlying skeletal changes.

Comparison of mandibular study models for sizechanges also indicated disproportional changes with narrowing in the canine region and widening in the premolar, molar and interalveolar areas in the test group. The decrease in length in the canine region may be explained by increased lip pressure affecting the lower arch as a consequence of a change in posture of the mandible. Extension of the head and an altered mode of breathing have also been reported to increase lip pressure (Solow and Sonnesen, 1998).

In the present study, statistically significant differences were found between the two groups for the measurements of palatal depth with the mean palatal depth in patients being greater. However the maxillary arch in patients was found to be wider in the premolar and molar regions with a narrowing of interalveolar width, measured at the level of first molars. These data suggest that the height of the palate, by itself is not a reliable indicator of maxillary constriction and that the width must also be taken into account when assessing constriction. From the findings of the study, it may be concluded that the palatal depth is more descriptive of constriction in the posterior part of the palate and that the shapes of the palate are quite different between the two groups.

The selection of non-obstructed controls was based on occlusal criteria, since the purpose of the study was to compare the occlusal variables of patients with sleepdisordered breathing and normal controls having an ideal occlusion.The prevalence of a Class II molar relationship was increased in this sample of OSA patients and a retrognathic mandible, which is often related to Class II malocclusions, has often been reported in conjunction with obstructed sleep and has been regarded as a predisposing factor for the development of OSA (Kawashima, Peltomäki, Sakata, Mori, Happonen, \& Rönning, 2002). The severity of OSA was also found to increasing in patients with severe Class II jaw relationship.

\section{Study limitations}

This is a cross-sectional study based on enrolment of patients attending few hospital clinics. Further research is necessary to generalise the results to the general population and other ethnicities. Another limitation of this study was that PSC was not carried out for the controls, due to ethical considerations and the prohibitive expense of the procedure.

\section{CONCLUSION}

The following conclusions can be drawn from this study:

- Indian adults with sleep disordered breathing have a dental arch morphology which is different from that of normal subjects, of similar age and gender.

- The prevalence of recorded malocclusions in the OSA group in terms of molar/canine relation is high, with $60 \%$ of the test subjects having an Angle's Class II malocclusion.

From these findings, it is evident that it is necessary to give due consideration to the evaluation of dental arch morphology of patients with sleep disordered breathing before the appropriate treatment is offered. The results of this study could facilitate the early recognition of the condition and support the available diagnostic setup. 


\section{References}

Battagel, J. M., Johal, A., \& Kotecha, B. (2000). A cephalometric comparison of subjects with snoring and obstructive sleep apnoea. The European Journal of Orthodontics, 22(4), 353-365.

Chervin, R. D., Murman, D. L., Malow, B. A., \& Totten, V. (1999). Cost-utility of three approaches to the diagnosis of sleep apnea: polysomnography, home testing, and empirical therapy. Annals of internal medicine, 130(6), 496-505.

Cistulli, P. A., Richards, G. N., Palmisano, R. G., Unger, G., Berthon-Jones, M., \& Sullivan, C. E. (1996). Influence of maxillary constriction on nasal resistance and sleep apnea severity in patients with Marfan's syndrome. CHEST Journal, 110(5), 11841188.

Dupont, W. D., Plummer, W. D. (1997). PS power and sample size program available for free on the internet. Controlled Clinical Trials. Available at: http://www.mc.vanderbilt.edu/prevmeed// ps.htm

Ferguson, K. A., Ono, T., Lowe, A. A., Ryan, C. F., \& Fleetham, J. A. (1995). The relationship between obesity and craniofacial structure in obstructive sleep apnea. Chest Journal, 108(2), 375381

Fleisher, K. E., \& Krieger, A. C. (2007). Current trends in the treatment of obstructive sleep apnea. Journal of Oral and Maxillofacial Surgery, 65(10), 2056-2068.

Harris, E. F., \& Johnson, M. G. (1991). Heritability of craniometric and occlusal variables: a longitudinal sib analysis. American Journal of Orthodontics and Dentofacial Orthopedics, 99(3), 258268.

Kawashima, S., Peltomäki, T., Sakata, H., Mori, K., Happonen, R. P., \& Rönning, O. (2002). Craniofacial morphology in preschool children with sleep-related breathing disorder and hypertrophy of tonsils. Acta paediatrica, 91(1), 71-77.

Lam, B., Ip, M. S. M., Tench, E., \& Ryan, C. F. (2005). Craniofacial profile in Asian and white subjects with obstructive sleep apnoea. Thorax, 60(6), 504-510.
Lee, R. W., Chan, A. S., Grunstein, R. R., \& Cistulli, P. A. (2009) Craniofacial phenotyping in obstructive sleep apnea-a novel quantitative photographic approach. Sleep, 32(1), 37-45.

Pirilá, K., Tahvanainen, P., Huggare, J., Nieminen, P., \& Löppönen, H. (1995). Sleeping positions and dental arch dimensions in children with suspected obstructive sleep apnea syndrome. European journal of oral sciences, 103(5), 285-291.

Revicki, D. A., \& Israel, R. G. (1986). Relationship between body mass indices and measures of body adiposity. American Journal of Public Health, 76(8), 992-994.

Sayin, M. O., \& Turkkahraman, H. (2004). Comparison of dental arch and alveolar widths of patients with Class II, division 1 malocclusion and subjects with Class I ideal occlusion. The Angle Orthodontist, 74(3), 356-360.

Solow, B., \& Sonnesen, L. (1998). Head posture and malocclusions. The European Journal of Orthodontics, 20(6), 685-693.

Thilander, B., Pena, L., Infante, C., Parada, S. S., \& de Mayorga, C. (2001). Prevalence of malocclusion and orthodontic treatment need in children and adolescents in Bogota, Colombia. An epidemiological study related to different stages of dental development. The European Journal of Orthodontics, 23(2), 153168.

Udwadia, Z. F., Doshi, A. V., Lonkar, S. G., \& Singh, C. I. (2004). Prevalence of sleep-disordered breathing and sleep apnea in middle-aged urban Indian men. American journal of respiratory and critical care medicine, 169(2), 168-173.

WJohns, M. (1993). Daytime sleepiness, snoring, and obstructive sleep apnea.Chest, 103, 30-36.

Zonato, A. I., Bittencourt, L. R., Martinho, F. L., Junior, J. F. S., Gregório, L. C., \& Tufik, S. (2003). Association of systematic head and neck physical examination with severity of obstructive sleep apnea-Hypopnea syndrome. The Laryngoscope, 113(6), 973-980. 